\title{
Markov Chain Model of HomePlug CSMA MAC for Determining Optimal Fixed Contention Window Size
}

\author{
Evan Kriminger ${ }^{*}$ and Haniph Latchman ${ }^{\dagger}$ \\ Dept. of Electrical and Computer Engineering, University of Florida, Gainesville, FL, USA \\ evankriminger@gmail.com* and latchman@qcslink.com ${ }^{\dagger}$
}

\begin{abstract}
This paper analyzes the optimum constant contention window (CW) for the HomePlug 1.0 and AV CSMA/CA MAC. A discrete time, homogenous Markov chain, with the state specified by both the backoff counter (BC) and deferral counter (DC), is used to model a single node contending for transmission. The structure of the Markov chain admits a generalized expression for the stationary state probability mass function (pmf) associated with each state. The recursively defined state pmfs can be analytically reduced to a single expression relating the probability $p$, of the node finding the medium idle, the maximum window size $W$, the maximum deferral counter size, and the number of nodes $n$. Optimizing the MAC efficiency provides a target value for $p$, which can be attained with the proper selection of $W$ and . It is shown that an optimal contention window size can be chosen based on a linear relationship with the number of nodes.
\end{abstract}

Keywords--HomePlug 1.0; HomePlug AV; contention window; CMSA-CA; medium access control

\section{INTRODUCTION}

The HomePlug 1.0 and HomePlug AV MAC protocols both use a carrier sense medium access with collision avoidance (CSMA-CA) scheme that offers several improvements over the IEEE 802.11 CSMA/CA protocol. When a node wishes to send, but detects the medium busy, it generates a backoff time, which is a uniform random variable between 0 and $W$-1, inclusive, where $W$ is the window size. This value decrements by one after every time slot and when it reaches zero, the node transmits. Another parameter, the deferral counter, allows the node to reduce the risk of collision when there is high traffic. The deferral counter is set to $\lambda$ when the backoff time is established. For every slot in which the medium is detected busy, the DC decrements by one. If the DC drops below zero, it is reset to a new, higher value and the backoff time is recalculated based on a new $W$ size that corresponds to the new $\lambda$. In this way, the node changes its backoff to accommodate a busier medium [3].

The MAC efficiency, $\eta$, of this system suffers a significant drop when the number of contending nodes, $n$ increases [1], as is expected to be the case as more and more communication and environmental monitoring and control devices are connected to the PLC grid. As the backoff times increase, the medium is used inefficiently, with efficiencies as low as 0.2 for 30-50 nodes. A modified MAC has been proposed that sets $W$ and $\lambda$ to constant values, which are selected based on $n$ to optimize the MAC efficiency.

\section{MODEL}

A discrete time, homogenous Markov chain shown in Fig. 1 is used to model a single node in a system of $n$ nodes. The assumption is that all nodes are contending for the medium, which establishes a "worst-case-scenario" for network traffic. The state pmf is a function of the backoff counter, $b$, and the deferral counter, $d$. It will be represented as $\Pi(d, b)$.

The probability that the node detects the medium idle is $p$ and also serves as the state transition probability between a node and the adjacent node one less in backoff count and of the same deferral count. The transition probability for nodes differing by one in both deferral count and backoff count is 1$p$. For nodes which have run the backoff counter to zero, the transition probability to any of the top row states (deferral counter $\lambda$ ) is $1 / W$. This transition probability corresponds to the random selection of the backoff time as described in the introduction. Finally, for states with a zero deferral count and nonzero backoff count, the transition probability to the top row states is $(1-p) / W$. This is similar to the previously calculated transition probability, but includes the chance of the deferral count dropping below zero when the medium is found to be busy.

The probability that the node transmits is $p_{0}$, and corresponds to the probability that the backoff counter has run to zero. It is given by the sum of all states in which $b$ is zero.

$$
p_{0}=\sum_{i=0}^{\lambda} \Pi(i, 0)
$$

Likewise, $p$ can be related to $p_{0}$, since the medium is detected idle when no other nodes are transmitting.

$$
p=\left(1-p_{0}\right)^{n-1}
$$

The object of this analysis is to eliminate the state pmfs and obtain a relation between $p$ and the parameters $W, \lambda$, and $n$.

\section{ANALYSIS}

The pmf for each state will be determined. This analysis will begin at the top row. For convenience, let $S_{\lambda}$ represent $\Pi(\lambda, W-1)$, the pmf of the rightmost top row state. We will show that all other state pmfs can be expressed as a function of $S_{\lambda}$. The $(\lambda, W-1)$ state arises from any of the $b=0$ states with probability $1 / W$ and from the $d=0, b \neq 0$ states with probability $(1-p) / W$. Therefore, $S_{\lambda}$ can be represented 


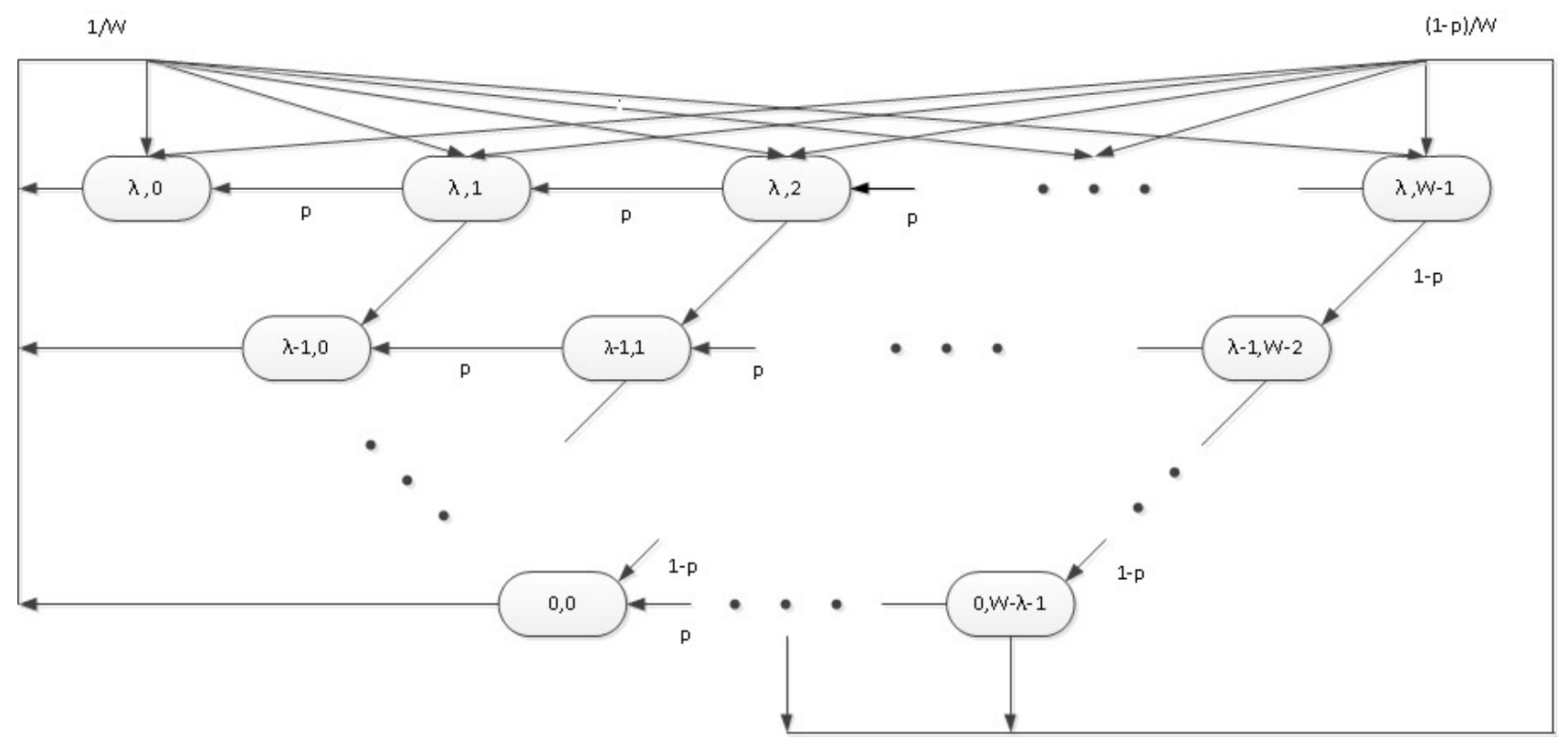

Figure 1 - Markov chain model for backoff procedure with fixed contention window.

$$
S_{\lambda}=\frac{1}{W} \sum_{i=0}^{\lambda} \Pi(i, 0)+\frac{1-p}{W} \sum_{j=1}^{W-\lambda-1} \Pi(0, j) .
$$

The other top row states have pmfs of the form

$$
\Pi(\lambda, b)=S_{\lambda}+p \Pi(\lambda, b+1),
$$

for all $0 \leq b \leq W-2$. We can remove the recursivity in $\Pi(\lambda, b)$ by tracing back to the initial $(\lambda, W-1)$ state. Then $\Pi(\lambda, b)$ is only a function of $S_{\lambda}$ and $p$ :

$$
\Pi(\lambda, b)=S_{\lambda} \sum_{i=0}^{W-1-b} p^{i},
$$

for all $0 \leq b \leq W-1$.

For the second row state pmfs, we will again start at the rightmost state of this row, $(\lambda-1, W-2)$. It can only be reached through the state $(\lambda, W-1)$, and hence has a probability given by

$$
\Pi(\lambda-1, W-2)=(1-p) S_{\lambda} .
$$

The next state to the left, $(\lambda-1, W-3)$, is reached either through the previous state of this row, $(\lambda-1, W-2)$, or from the state $(\lambda, W-2)$ on the higher row.

$$
\begin{gathered}
\Pi(\lambda-1, W-3)=p \Pi(\lambda-1, W-2)+(1-p) \Pi(\lambda, W-2) \\
=p(1-p) S_{\lambda}+(1-p) S_{\lambda}(p+1) \\
=(2 p+1)(1-p) S_{\lambda}
\end{gathered}
$$

Using the same method, the whole row can be determined.

$$
\begin{gathered}
\Pi(\lambda-1, W-4)=\left(3 p^{2}+2 p+1\right)(1-p) S_{\lambda} \\
\Pi(\lambda-1, W-5)=\left(4 p^{3}+3 p^{2}+2 p+1\right)(1-p) S_{\lambda}
\end{gathered}
$$

Each state $(d, b)$ depends on $(d, b+1)$ and $(d+1, b+1)$.

$$
\Pi(d, b)=p \Pi(d, b+1)+(1-p) \Pi(d+1, b+1)
$$

for $0 \leq d \leq \lambda-1$.

It turns out that this recursive structure yields state pmfs which can be represented in terms of polynomials in $p$, powers of $1-p$, and $S_{\lambda}$. Furthermore, the coefficients of the polynomials in $p$ for each row are diagonal elements of Pascal's triangle. Pascal's triangle elements are represented simply with the binomial coefficient and therefore we can write

$$
\Pi(d, b)=S_{\lambda}(1-p)^{\lambda-d} \sum_{i=0}^{W-\lambda-1+d-b}\left(\begin{array}{c}
\lambda-d+i \\
i
\end{array}\right) p^{i}
$$

for $0 \leq d \leq \lambda$.

These equations are still recursive because they rely on $S_{\lambda}$. However, (4) is linear in $S_{\lambda}$ and if we can factor out $S_{\lambda}$ and equate it to (3) it can be eliminated. A non-recursive solution for $S_{\lambda}$ is admitted by (4) using the normalization condition,

$$
1=\sum_{i=0}^{\lambda} \sum_{j=0}^{W-\lambda-1+i} \Pi(i, j)
$$

It will be easier to construct this sum from sums of the individual rows, because the pmfs of each state in a row share a similar form. This sum, which is a function of the deferral count of the row in question, will be represented by $R(d)$.

$$
R(d)=\sum_{i=0}^{W-\lambda-1+d} \Pi(d, i)
$$

for $0 \leq \mathrm{d} \leq \lambda$.

Substituting (4) into (6), yields (7). 


$$
\begin{aligned}
& R(d)= \\
& \sum_{i=0}^{W-\lambda-1+d} S_{\lambda}(1-p)^{\lambda-d} \sum_{j=0}^{W-\lambda-1+d-i}\left(\begin{array}{c}
\lambda-d+j \\
j
\end{array}\right) p^{j} \\
& =S_{\lambda}(1-p)^{\lambda-d} \sum_{i=0}^{W-\lambda-1+d}(W-\lambda+d-i)\left(\begin{array}{c}
\lambda-d+i \\
i
\end{array}\right) p^{i}
\end{aligned}
$$

Since the polynomial component of the pmf for all states in a row have the same coefficients, a term $p^{i}$ will be repeated exactly one time more than the next highest power $p^{i+1}$ in the sum and this is captured by the coefficient inside the summation of (7).

With this new representation of a row sum, (5) can be expressed

$$
1=\sum_{i=0}^{\lambda} R(i)
$$

We can factor out $S_{\lambda}$ from the sum in (8) and solve for $S_{\lambda}$.

$$
S_{\lambda}=\frac{1}{\sum_{i=0}^{\lambda} \frac{R(i)}{S_{\lambda}}}
$$

Between (3) and (9), the pmf $S_{\lambda}$ can be eliminated, leaving us with an expression relating only the desired parameters. However, (3) must be simplified; it contains sums of state pmfs. The first sum in (3) can be eliminated by employing (1), which defines $p_{0}$.

$$
S_{\lambda}=\frac{1}{W} p_{0}+\frac{1-p}{W} \sum_{j=1}^{W-\lambda-1} \Pi(0, j)
$$

Using (2), $p_{0}$ can be expressed in terms of $p$.

$$
S_{\lambda}=\frac{1}{W}\left(1-p^{\frac{1}{n-1}}\right)+\frac{1-p}{W} \sum_{j=1}^{W-\lambda-1} \Pi(0, j)
$$

The second sum in (3) can be expressed in terms of $R(d)$.

$$
\begin{gathered}
\sum_{j=1}^{W-\lambda-1} \Pi(0, j)=R(0)-\Pi(0,0) \\
=S_{\lambda}(1-p)^{\lambda} \sum_{i=0}^{W-\lambda-1}(W-\lambda-i-1)\left(\begin{array}{c}
\lambda+i \\
i
\end{array}\right) p^{i}
\end{gathered}
$$

Substituting (11) into (10) and solving for $S_{\lambda}$, we get

$$
\begin{aligned}
& S_{\lambda}= \\
& \frac{1-p^{\frac{1}{n-1}}}{W-(1-p)^{\lambda+1} \sum_{i=0}^{W-\lambda-1}(W-\lambda-i-1)\left(\begin{array}{c}
\lambda+i \\
i
\end{array}\right) p^{i}} .
\end{aligned}
$$

There are now two independent equations for $S_{\lambda}$, which are functions of $W, \lambda, p$, and $n$. Equating (9) and (12) and inverting for clarity, completely removes the state dependency.

$$
\begin{gathered}
\frac{W-(1-p)^{\lambda+1} \sum_{i=0}^{W-\lambda-1}(W-\lambda-i-1)\left(\begin{array}{c}
\lambda+i \\
i
\end{array}\right) p^{i}}{1-p^{\frac{1}{n-1}}} \\
=\sum_{i=0}^{\lambda} \frac{R(i)}{S_{\lambda}}
\end{gathered}
$$

Substituting (7), the right side of (13) becomes

$$
=\sum_{i=0}^{\lambda}(1-p)^{\lambda-i} \sum_{j=0}^{W-\lambda-1+i}(W-\lambda+i-j)\left(\begin{array}{c}
\lambda-i+j \\
j
\end{array}\right) p^{j}
$$

Equation (13) provides an analytical relationship between $W, \lambda$, $n$, and $p$.

\section{APPROXIMATION OF NODE TRANSMISSION PROBABILITY}

In [2], the probability of a node transmitting is approximated as

$$
p_{0} \approx \frac{2}{2 W-\lambda}
$$

which was calculated from the ratio of states which have a backoff counter value of zero. Notice that this solution is constant in $n$. A key contribution of the present paper is to obtain the exact analytical solution by solving for $p$ in (13) and using (2) to obtain $p_{0}$. This solution is dependent on $n$, unlike the approximation. For a given value of $W$ and $\lambda$, the approximation in (14) approaches the analytical solution for large $n$. In Fig. 2, the two $p_{0}$ values are plotted against $n$ for a fixed value of $W$ and $\lambda$. The magnitude of this error is quite severe for lower numbers of nodes.

The general shape of Fig. 2 is representative of the $p_{0}$ versus $n$ curve for all values of $W$ and $\lambda$. When these two parameters are changed, the error between the analytical and approximate solutions changes as well.

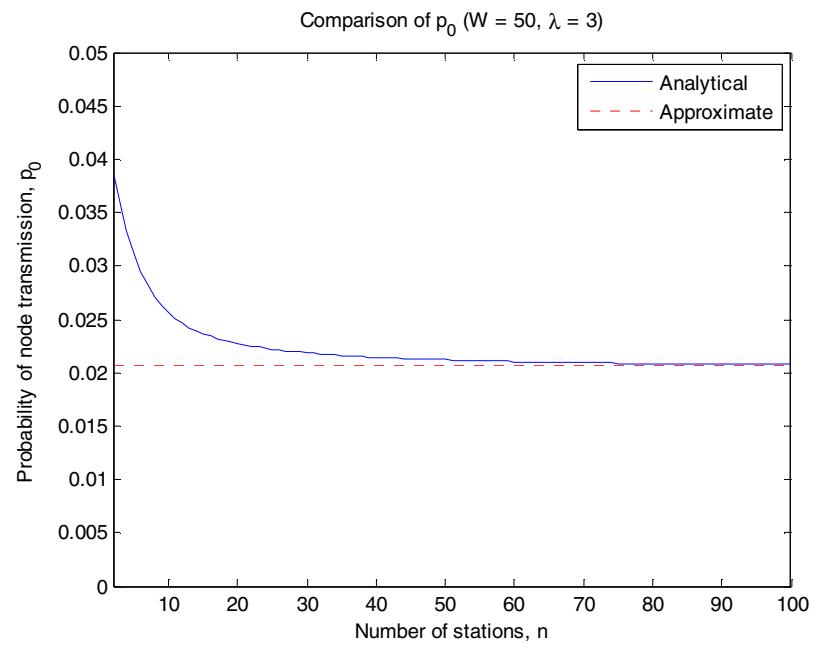

Figure 2 - Comparison of $p_{0}$ generated from the analytical and approximate solutions. 


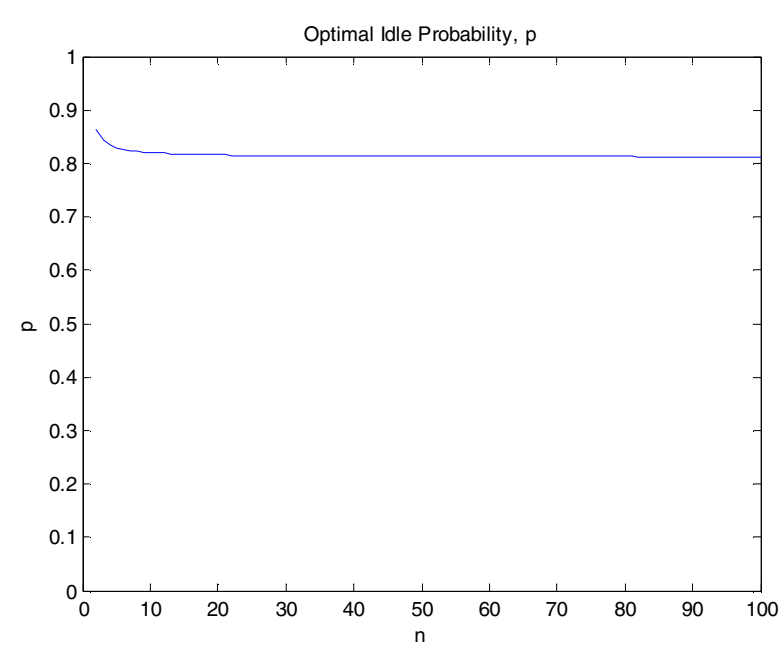

Figure 3-Optimal probability of finding the medium idle [2].

\section{OPTIMAL CONTENTION WINDOW}

Our parameters will be optimized in the sense of maximizing the MAC efficiency, as was performed in [2]. Let $P_{S}$ be the probability of successful transmission, $P_{I}$ be the probability of idle passage, and $P_{C}$ be the collision probability. In terms of $p_{0}$ these are

$$
\begin{gathered}
P_{S}=n p_{0}\left(1-p_{0}\right)^{n-1} \\
P_{I}=\left(1-p_{0}\right)^{n-1} \\
P_{C}=1-P_{S}-P_{I} .
\end{gathered}
$$

The MAC efficiency can be approximated by

$$
\eta=\frac{P_{S} T_{\text {Data }}}{P_{S} T_{S}+P_{C} T_{C}+P_{I} T_{I}}
$$

where $T_{S}, T_{C}$, and $T_{I}$ are the times of successful transmission, collision, and idle slot passage, respectively. The MAC efficiency can be optimized in $p_{0}$ for a given $n$. The result for this optimization given by [2] is

$$
1-\frac{T_{I}}{T_{C}}=\frac{1-n p_{0, o p t}}{\left(1-p_{0, o p t}\right)^{n}} .
$$

The corresponding optimal value of $p$ can be found using (2). Therefore, for a given $n$ and the value of $p_{\text {opt }}$ associated with it through (16), the values of $W$ and $\lambda$ which best satisfy (13) are optimal in the sense of MAC efficiency.

\section{RESULTS}

In determining the optimal $p_{0}$ from (16), the values of $T_{I}$ and $T_{C}$ will be set to 20 and $800 \mu \mathrm{s}$, respectively, as was done in [2]. The optimal parameters were found in [2] by substituting (14) into (16) and conducting a search for the best $W$ and $\lambda$. In particular, the optimal contention window is obtained as a function of $n$ when the value of $\lambda$ was fixed at 3 and 15. The result was an affine relationship between $W$ and $n$ with a slope

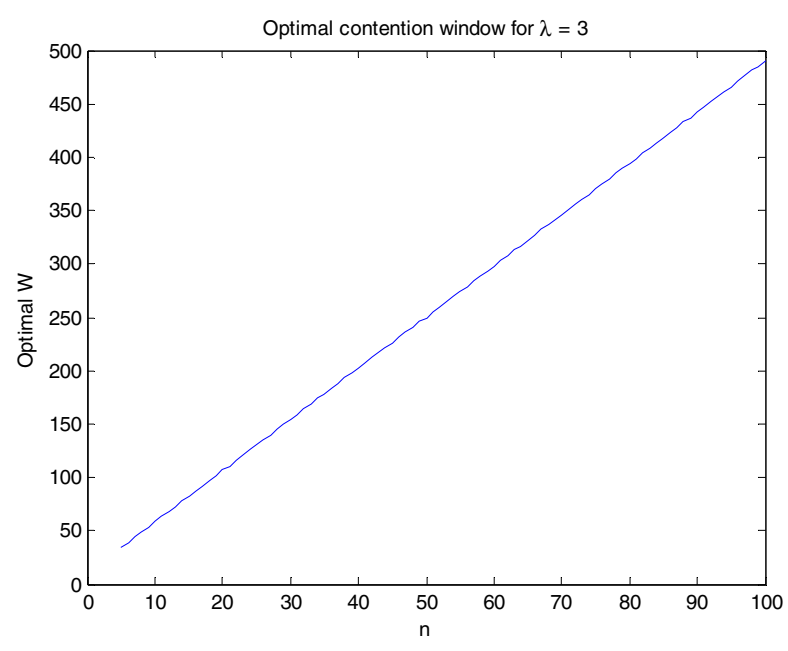

Figure 4 - Optimal contention window as a function of the number of stations for $\lambda=3$.

that remains constant for all $\lambda$, and an intercept that changes with $\lambda$. For example,

$$
W \approx 5 n+10 \text { for } \lambda=3,
$$

and

$$
W \approx 5 n+35 \text { for } \lambda=15 \text {. }
$$

The analytical solution reveals the true relationship. To find the optimal parameters, we search through the reasonable values of $\lambda$ and $W$. For a given $n$, each combination of these parameters will yield a value of $p$ that solves (13). The combination of $W$ and $\lambda$ that yields $p$ nearest to the optimal value will be optimal. If a set of $\lambda$ and $W$ are optimal for a given $n$, then the $p$ that results from (13) must be within $1 \%$ of the optimal value. First, for comparison with [2] this search will be conducted by finding the optimal $W$ for a predetermined $\lambda$.

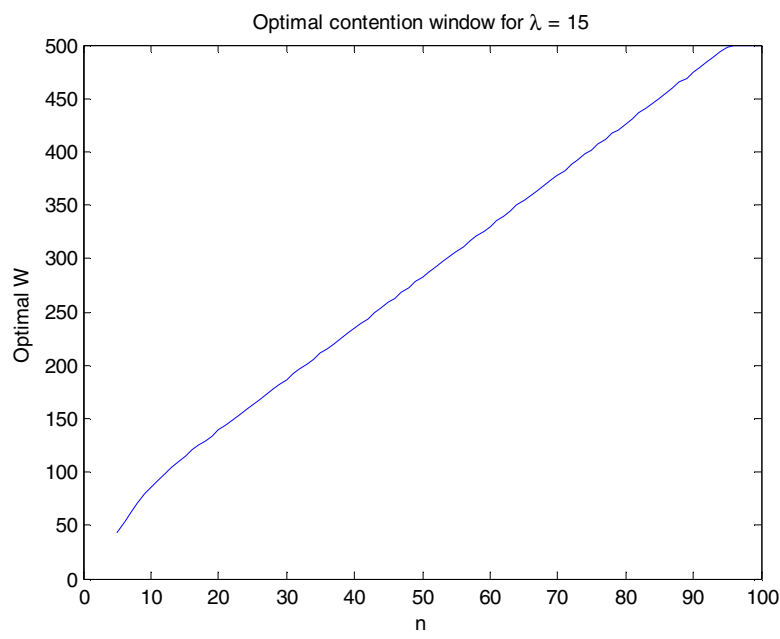

Figure 5 - Optimal contention window for $\lambda=15$. 


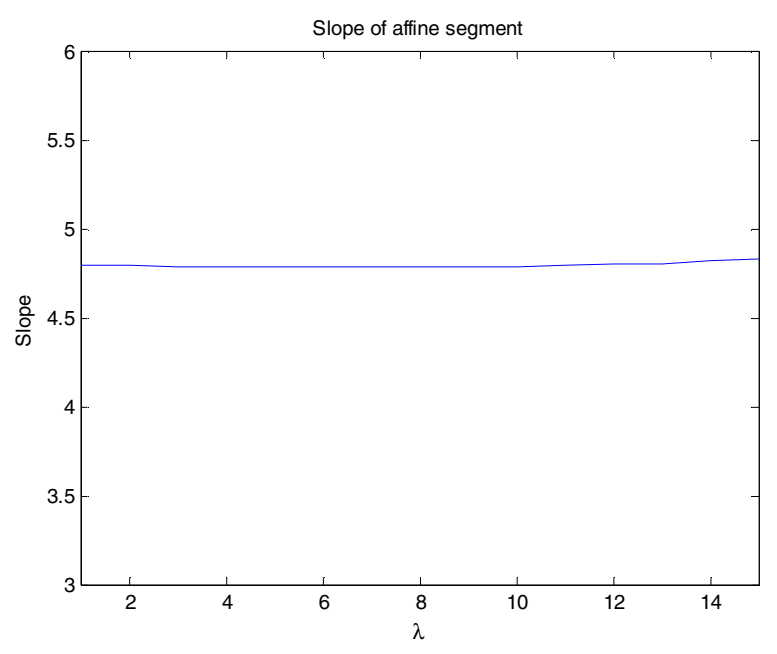

Figure 6 - Slope of the best-fit line as a function of $\lambda$.

For sufficiently large $n$, the relationship between $W$ and $n$ is affine as in [2], but it is nonlinear for low $n$. The number of nodes at which linearity can be assumed increases proportionally to $\lambda$. Since our work revolves around the MAC efficiency at high $n$, linearity can be assumed for reasonable values of $\lambda$. For comparison, the analytical result yielded the following relationships for the affine segment,

$$
W \approx 4.8 n+10.8 \text { for } \lambda=3,
$$

and

$$
W \approx 4.8 n+41.5 \text { for } \lambda=15 .
$$

In Fig. 4, we have an example of a lower $\lambda$, where the optimal $W$ relates to $n$ linearly. In Fig. 5 is an example of a higher $\lambda$, where the linear relation fails for small $n$. For $\lambda=15$, we can assume linearity for $n>10$.

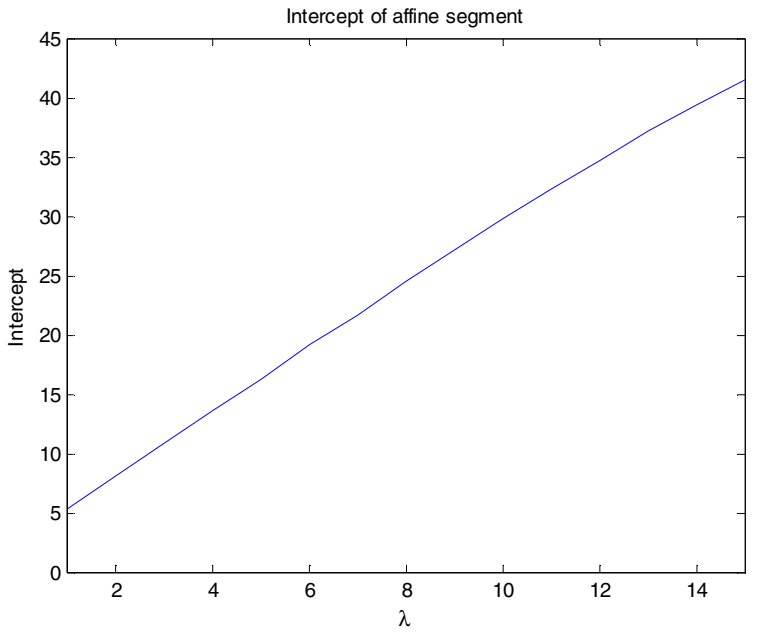

Figure 7 - Intercept of best-fit line as a function of $\lambda$.

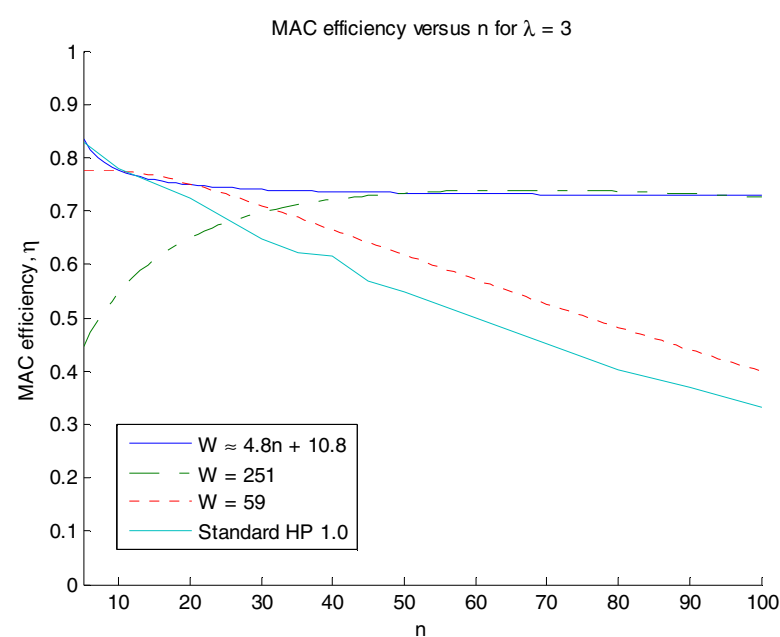

Figure 8-MAC efficiency as a function of $n$ for three choices of $W$, compared with the standard HomePlug 1.0 MAC efficiency.

The parameters of the best-fit line for the linear segment of the $n-W$ curve can be easily visualized by plotting them against a changing $\lambda$. From Fig. 6 , the slope remains approximately constant for all $\lambda$. In Fig. 7 it is apparent that the intercept increases nearly linearly with $\lambda$. The choice of $\lambda$ is not significant. We simply select a reasonable value, and then have the optimal $W$ as an affine function of $n$.

In Fig. 8 we show the benefits of adaptively selecting the optimal window size for a given $n$. For the purposes of comparison, we also show the MAC efficiency for the standard HomePlug 1.0 CSMA/CA protocol. In this plot, $\lambda$ was set to 3 . Three different selection schemes for $W$ are shown. We first change $W$ to the optimal value for each $n$, which is approximately $4.8 n+10.8$ for $\lambda=3$. Then $W$ is fixed at 251 and 59, which are its optimal values at $n=50$ and $n=10$, respectively. The MAC efficiency is determined from (15), using $T_{S}=1100 \mu \mathrm{s}$ and $T_{\text {Data }}=1000 \mu \mathrm{s}$, which are reasonable values in accordance to the protocol [3]. By adapting $W$ with $n$, we maintain a high MAC efficiency. Any fixed value of $W$ will suffer serious degradations in efficiency for some or all values of $n$.

\section{CONCLUSIONS}

The Markov chain model for HomePlug 1.0 and AV CSMA/CA backoff has a solution free of recursion. Therefore, approximations can be avoided in the optimum contention window calculation. The optimum contention window calculation provided by [2] uses a simplified probability of node transmission, which approaches the analytical value only for large $n$. This approximate solution generates an optimum contention window for a given $\lambda$ that can be calculated as an affine function of $n$ with a constant slope. This lends itself to easy adaptation using node estimation strategies.

The analytical solution determines that for a given $\lambda$, the contention window is an affine function of $n$, as long as $n$ is past a threshold value. The threshold however, is small for 
values of $\lambda$ consistent with the current HomePlug protocol. The number of nodes at which MAC efficiency drops should exceed this value, so linearity is a good assumption for the cases of interest.

The drop in MAC efficiency with a large number of contending stations can now be avoided by estimating $n$, and then updating the backoff window accordingly. Only a single slope and intercept need be stored, and the optimum contention window can be generated for all $n$.

\section{REFERENCES}

[1] K. Tripathi, J. D. Lee, H. A. Latchman, and J. McNair, "Contention window based parameter selection to improve powerline MAC efficiency for large number of users," accepted for publication in IEEE Int. Sym. on Power Line Communications and its Applications, March 2006.
[2] J. D. Lee, K. Tripathi, H. A. Latchman, and J. McNair, "Population based adaptive tuning of constant contention window for HomePlug 1.0," Int. J. of Power and Energy Systems, Vol. 203-4065, pp. 192-205, 2008

[3] M. K. Lee, R. E. Newman, H. A. Latchman, S. Katar, and L. Yonge, "HomePlug 1.0 powerline communication LANs - protocol description and performance results," Int. J. of Communication Systems, 2003.

[4] A. Leon-Garcia, Probability, Statistics, and Random Processes for Electrical Engineering, Prentice Hall, $3^{\text {rd }}$ ed., 2008.

[5] F. Cali, M. Conti, E. Gregori, "Dynamic tuning of the IEEE 802.11 protocol to achieve a theoretical throughput limit," IEEE/ACM Trans. On Networking 8 (6) (2000) 785-799.

[6] G. Bianchi, "Performing analysis of the IEEE 802.11 distributed coordination function," IEEE J. on Selected Areas in Communication, 18(3), March 2000, 535-547.

[7] F. Cali, M. Conti, and E. Gregori, "IEEE 802.11 protocol: design and performance evaluation of an adaptive backoff mechanism," CNUCE Internal Report, March 2000. 